Pol. J. Food Nutr. Sci., 2021, Vol. 71, No. 4, pp. 403-410

On-line ISSN: 2083-6007

Print ISSN: $1230-0322$

DOI: $10.31883 /$ pjfns/142879 http://journal.pan.olsztyn.pl

Original article

Section: Food Chemistry

\title{
Non-Destructive Quantitative Analysis of Azodicarbonamide Additives in Wheat Flour by High-Throughput Raman Imaging
}

\author{
Xiaobin Wang ${ }^{1,2,3,4,5 * \odot \text {, Chunjiang Zhao }}$, 3, 4,5 $^{\circ}$ \\ ${ }^{I}$ School of Physics and Electronic Information, Nanchang Normal University, Nanchang 330032, China \\ ${ }^{2}$ Beijing Research Center of Intelligent Equipment for Agriculture, Beijing 100097, China \\ ${ }^{3}$ National Research Center of Intelligent Equipment for Agriculture, Beijing 100097, China \\ ${ }^{4}$ Key Laboratory of Agri-informatics, Ministry of Agriculture, Beijing 100097, China \\ ${ }^{5}$ Beijing Key Laboratory of Intelligent Equipment Technology for Agriculture, Beijing 100097, China
}

Key words: azodicarbonamide, wheat flour, Raman imaging, image classification, quantitative model

Azodicarbonamide (ADA) additives are limited or prohibited from being added to wheat flour by various countries because they may produce carcinogenic semicarbazide in humid and hot conditions. This study aimed to realize the non-destructive detection of ADA additives in wheat flour using high-throughput Raman imaging and establish a quantitative analysis model. Raman images of pure wheat flour, pure ADA, and wheat flour-ADA mixed samples were collected respectively, and the average Raman spectra of each sample were calculated. A partial least squares (PLS) model was established by using the linear combination spectra of pure wheat flour and pure ADA and the average Raman spectra of mixed samples. The regression coefficients of the PLS model were used to reconstruct the 3D Raman images of mixed samples into 2D grayscale images. Threshold segmentation was used to classify wheat flour pixels and ADA pixels in grayscale images, and a quantitative analysis model was established based on the number of ADA pixels. The results showed that the minimum detectable content of ADA in wheat flour was $100 \mathrm{mg} / \mathrm{kg}$. There was a good linear relationship between the ADA content in the mixed sample and the number of pixels classified as ADA in the grayscale image in the range of $100-10,000 \mathrm{mg} / \mathrm{kg}$, and the correlation coefficient was 0.9858 . This study indicated that the combination of PLS regression coefficients with threshold segmentation had provided a non-destructive method for quantitative detection of ADA in Raman images of wheat flour-ADA mixed samples.

\section{INTRODUCTION}

Wheat is the staple food for more than one-third of the world's population. Flour is the main product of wheat processing, which is often used to make steamed buns, noodles, bread, instant noodles, dumplings and other kinds of food. The wheat flour quality differs due to the effects of wheat varieties, growth environment, storage and transportation, processing methods and other factors [Lancelot et al., 2021; Liu et al., 2015; Lv et al., 2013]. To meet the market demand for different wheat flour products, the company needs to add various types of additives in the course of processing. The use of additives is directly related to the wheat flour quality and people's dietary safety.

As a wheat flour quality improver, azodicarbonamide (ADA) is used to increase the strength and flexibility of dough and has a certain bleaching effect [Yasui et al., 2016]. ADA acts as an oxidizing agent when wheat flour is stirred into dough with water, and may be transformed into semicarbazide after heat treatment [Becalski et al., 2004; Ye et al., 2011]. Some experiments have shown the potential carcinogenicity

\footnotetext{
* Corresponding Author:

E-mail: tawangxiaobin@126.com (X.Wang)
}

of semicarbazide in animals [Tian et al., 2014]. Therefore, the international restrictions on the use of ADA vary. The European Union, Australia, New Zealand, Singapore, and Japan have all banned the use of ADA in food, but it can be used as a wheat flour additive in the United States, Brazil, Canada, and China at the maximum dose of $45 \mathrm{mg} / \mathrm{kg}$ [Chen et al., 2018].

At present, the conventional detection methods of ADA in wheat flour are mainly high-performance liquid chromatography (HPLC) [Li et al., 2015; Wei et al., 2017] and liquid chromatography-mass spectrometry (LC-MS) [Noonan et al., 2005; Wang et al., 2014]. These two chromatographic methods can accurately detect the ADA content in wheat flour, and have the advantages of low detection limit, strong specificity, and high sensitivity. However, they require a series of complex pretreatments, and the selection of chromatographic conditions, such as chromatography column, flow rate of carrier gas, and sample size, also needs to be taken into account. The entire operation process requires skilled operators and has complicated, time- and cost-consuming, and destructive procedures. 
As the optoelectronic technology develops, some spectroscopy methods have been shown reliable in the detection of wheat flour additives, such as fluorescence spectroscopy [Chen et al., 2011], Raman spectroscopy [Cebi et al., 2017], near-infrared spectroscopy [Che et al., 2017; Gao et al., 2016], and terahertz spectroscopy [Hu et al., 2020; Sun et al., 2019]. However, these spectral methods only obtain the information of a single sampling point, which poses the problem of sampling representativeness. In addition, only the spectral signal of the sample can be obtained, and the distribution of the substance inside the sample cannot be known. High-throughput Raman imaging integrates the advantages of Raman spectroscopy and digital imaging, which can obtain Raman spectra and spatial distribution information simultaneously during sample scanning [Qin et al., 2010; Wang et al., 2017a; Zhai et al., 2017]. There are three ways to acquire Raman images: point-scan, line-scan, and plane-scan [Lohumi et al., 2017]. Among them, the detection speed of line-scan mode is faster than that of the point-scan, which can be applied to the detection of samples with flat surface, and shows a good prospect to be used for powdered food safety assessment. Currently, the related reports on the detection of wheat flour additives by line-scan Raman imaging focus on benzoyl peroxide (BPO) [Li et al., 2019; Qin et al., 2017; Wang et al., 2017b]. All these reports provided the spatial distribution and quantitative detection model of BPO in wheat flour, but the data processing method is achieved by selecting the grayscale image corresponding to the Raman peak with the highest intensity in the additive Raman spectrum combined with the threshold segmentation method, ignoring the influence of other bands in the Raman spectrum of the additive on the detection results. In this way, in order to consider the influence of each band of the Raman spectrum on the detection of ADA in wheat flour, we applied the regression coefficients of the partial least squares (PLS) model to all the bands of the sample Raman spectrum to enable the quantitative analysis of ADA in wheat flour.

This study aimed to achieve the non-destructive quantitative analysis of ADA additives in wheat flour by high-throughput Raman imaging. Specific objectives were to: 1) acquire Raman images of wheat flour, ADA, and wheat flour-ADA mixed samples, and find the Raman characteristic peaks of wheat flour and ADA; 2) establish a PLS model and extract the regression coefficients to reconstruct the Raman image into a grayscale image; and 3) create a binary image to classify wheat flour pixels and ADA pixels, and establish a quantitative analysis model for ADA detection in wheat flour.

\section{MATERIALS AND METHODS}

\section{Instruments and reagents}

The high-throughput Raman imaging system was assembled by Isuzu Optics Corp. (Shanghai, China), and its detailed description can be found in Wang et al. [2017a]. Electronic balance (FA2204B) was obtained from Shanghai Precision and Scientific Instrument Corp. (Shanghai, China), with a weighing range of $0-220 \mathrm{~g}$ and an accuracy of $0.1 \mathrm{mg}$. Vortex Mixer (Vortex-Genie 2) was purchased from Scientific Industries Inc. (New York, NY, USA), with a 600-3,200 rpm speed range. Customized square aluminum alloy container had the internal size of $45 \times 45 \times 2 \mathrm{~mm}$ (we have determined in the previous study that the effective penetration depth of line laser to wheat flour is $2 \mathrm{~mm}$ ).

ADA (97\%) was purchased from Xiya Chemical Technology Co. Ltd. (Linyi, China). Wheat flour was obtained from a local supermarket in Beijing (China), and was determined by HPLC to be ADA free.

\section{Sample preparation}

The electronic balance was used to accurately weigh $0.1 \mathrm{~g}$ of ADA and $9.9 \mathrm{~g}$ of wheat flour, that were transferred into a $50 \mathrm{~mL}$ centrifuge tube and mixed evenly with a vortex mixer to obtain a wheat flour-ADA mixed sample containing 10,000 mg of ADA per $\mathrm{kg}$ of wheat flour. The mass of ADA and wheat flour was adjusted to keep the total mass of each mixed sample at $10 \mathrm{~g}$, and the mixed samples with 9 different ADA contents in wheat flour $(100 ; 200 ; 500 ; 800$; 1,$000 ; 2,000 ; 5,000 ; 8,000 ;$ and $10,000 \mathrm{mg} / \mathrm{kg}$ ) were prepared. The mass of the mixed sample in the square container was about $2.1 \mathrm{~g}$. To make full use of the mixed samples, each mixed sample was put into four identical square containers to obtain four subsamples. Meanwhile, the pure wheat flour sample and pure ADA sample were prepared and divided into four subsamples for Raman image acquisition.

\section{Raman image collection}

The sample was placed on a single-axis moving platform, and the height was adjusted to ensure that the sample surface was $20 \mathrm{~cm}$ away from the lens. The exposure time of the camera was $1,000 \mathrm{~ms}$, and the spatial resolution was $0.125 \mathrm{~mm} /$ pixel. The Raman spectrum collection range was 785-1,000 nm (corresponding to the Raman shift was $0-2,728 \mathrm{~cm}^{-1}$ ), and the spectral resolution was $0.54 \mathrm{~nm}$. The moving speed of the single-axis moving platform was $0.0823 \mathrm{~mm} / \mathrm{s}$. The acquisition area of the camera was $128 \times 45 \mathrm{~mm}$ (spatial information was $1,024 \times 360$ pixels), and the data was saved as 1,024 pixels $\times 360$ pixels $\times 512$ wavelengths Raman image cube.

\section{Data processing}

To reduce the data volume and quickly extract target information, it was necessary to determine the region of interest (ROI) and spectral range of the Raman image. The center of the sample Raman image was taken as the center of ROI, and a $40 \times 40 \mathrm{~mm}$ square area (corresponding to $320 \times 320$ pixels, a total of 102,400 pixels) was selected to ensure that only the sample area was included and the background area was excluded. The Raman peak of ADA was mainly located in 400-2000 $\mathrm{cm}^{-1}$, and this spectral range was selected for subsequent analysis. The mixed sample was irradiated by laser to generate Raman signals and accompanied by fluorescence signals, and the high fluorescence directly interfere with the identification of Raman peak. The adaptive iteratively reweighted penalized least squares method can fit the Raman spectrum to eliminate the interference of the background signal, so this method was selected for the correction of the Raman spectrum [Zhang et al., 2010].

The PLS model was established using the ADA content and Raman spectra of wheat flour-ADA mixed samples, 
in which the calibration set was the average Raman spectra of mixed samples, and the prediction set was the new Raman spectrum obtained by combining the Raman spectrum of wheat flour and ADA. The new Raman spectrum as given in equation (1):

$$
S_{i}^{n e w}=S_{a} C_{i}+\frac{S_{f}}{1-C_{i}}
$$

where: $S_{i}^{\text {new }}$ is the new Raman spectrum; $S_{a}$ is the Raman spectrum of ADA; $S_{f}$ is the Raman spectrum of wheat flour; $C_{i}$ is the ADA content of the mixed sample; a total of 9 ADA contents $(100 ; 200 ; 500 ; 800 ; 1,000 ; 2,000 ; 5,000 ; 8,000$; $10,000 \mathrm{mg} / \mathrm{kg}$ ) was used.

The coefficient of determination (calibration set is $R_{c}^{2}$, prediction set is $R_{p}^{2}$ ) and root mean square error (RMSE) (calibration set is RMSEC; prediction set is RMSEP) was used to evaluate the performance of the PLS model. The regression coefficient of the optimal PLS model was extracted and used to calculate the intensity of each pixel in the Raman image of the mixed sample. The intensity of each pixel was computed according to equation (2):

$$
I=R_{1} X_{1}+R_{2} X_{2}+\ldots \ldots+R_{\mathrm{n}} X_{\mathrm{n}}+R_{0}
$$

where: $I$ is the intensity; $R_{1}-R_{\mathrm{n}}$ are the regression coefficients of each band in the PLS model; $X_{1}-X_{\mathrm{n}}$ are the Raman intensity of each band in the Raman spectrum of the mixed sample; $R_{0}$ is the intercept.

To obtain the PLS model with high predictive performance, different methods were used to preprocess the Raman spectra of the calibration set and the prediction set, including normalization, and multiplicative scatter correction (MSC), standard normal variate transformation (SNV), first derivative $\left(1^{\text {st }}\right)$, and second derivative $\left(2^{\text {nd }}\right)$.

The threshold segmentation method was used to create binary images to classify ADA pixels and wheat flour pixels in grayscale images of mixed samples, and the threshold was determined by the maximum gray value in the grayscale image of pure wheat flour. Those with gray values above and below the threshold were classified as ADA pixels and wheat flour pixels, respectively. PLS model establishment and spectral pretreatment were performed by The Unscrambler X10.4 Software (Camo Software AS, Oslo, Norway), and the rest of the process was completed using MATLAB 7.11 program (Math Works Inc., Natick, MA, USA).

\section{RESULTS AND DISCUSSION}

\section{Average Raman spectra of wheat flour and ADA}

The average Raman spectra of wheat flour and ADA are shown in Figure 1. The Raman spectra of the two are significantly different. There was a high fluorescence background in the Raman spectrum of wheat flour (Figure 1a), which causes the baseline to drift. The peak with the highest intensity was located at $481 \mathrm{~cm}^{-1}$ in the corrected Raman spectrum, which was consistent with the results obtained by other researchers [Czaja et al., 2016]. This Raman peak was assigned to the coupling of $\mathrm{C}-\mathrm{C}-\mathrm{C}$ skeleton bending vibration and C-O deformation vibration [Wiercigroch et al., 2017].

The Raman spectrum of ADA had a flat baseline and many Raman peaks (Figure 1b). Three obvious peaks could be observed at 1,$121 ; 1,335$; and $1,577 \mathrm{~cm}^{-1}$, respectively. Among them, the $1,335 \mathrm{~cm}^{-1}$ peak had the highest intensity. The model of the molecular structure of ADA with $\mathrm{C}-\mathrm{N}$, $\mathrm{C}=\mathrm{O}, \mathrm{N}=\mathrm{N}, \mathrm{N}-\mathrm{H}$ and other bonds is shown in Figure $1 \mathrm{~b}$ (upper left diagram). Its molecular formula is $\mathrm{C}_{2} \mathrm{H}_{4} \mathrm{~N}_{4} \mathrm{O}_{2}$. Each bond of the functional group has its characteristic vibration frequency, and the Raman peaks can be assigned according to the different vibration frequencies. The wavenumber of $1,121 \mathrm{~cm}^{-1}$ was assigned to the in-plane bending vibration of $\mathrm{H}-\mathrm{N}-\mathrm{H} ; 1,335 \mathrm{~cm}^{-1}$ was assigned to the asymmetric stretching vibration of $\mathrm{N}-\mathrm{C}-\mathrm{N}$, which was also accompanied by the in-plane bending vibration of $\mathrm{N}-\mathrm{H}$; and $1,577 \mathrm{~cm}^{-1}$ was assigned to the $\mathrm{N}=\mathrm{N}$ stretching vibration [Li et al., 2015; Xie et al., 2013].
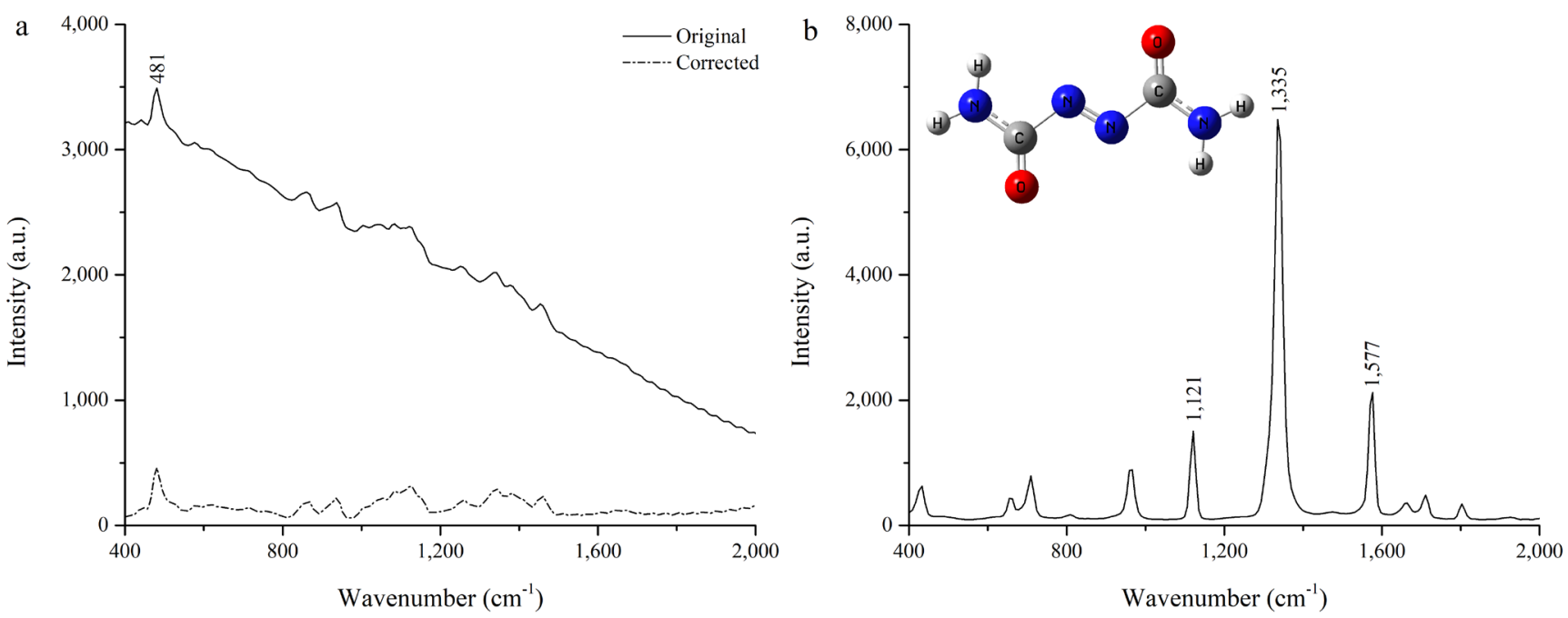

FIGURE 1. Average Raman spectra of (a) wheat flour and (b) azodicarbonamide (ADA). ADA structure model is presented in left upper corner of Figure 1b. 


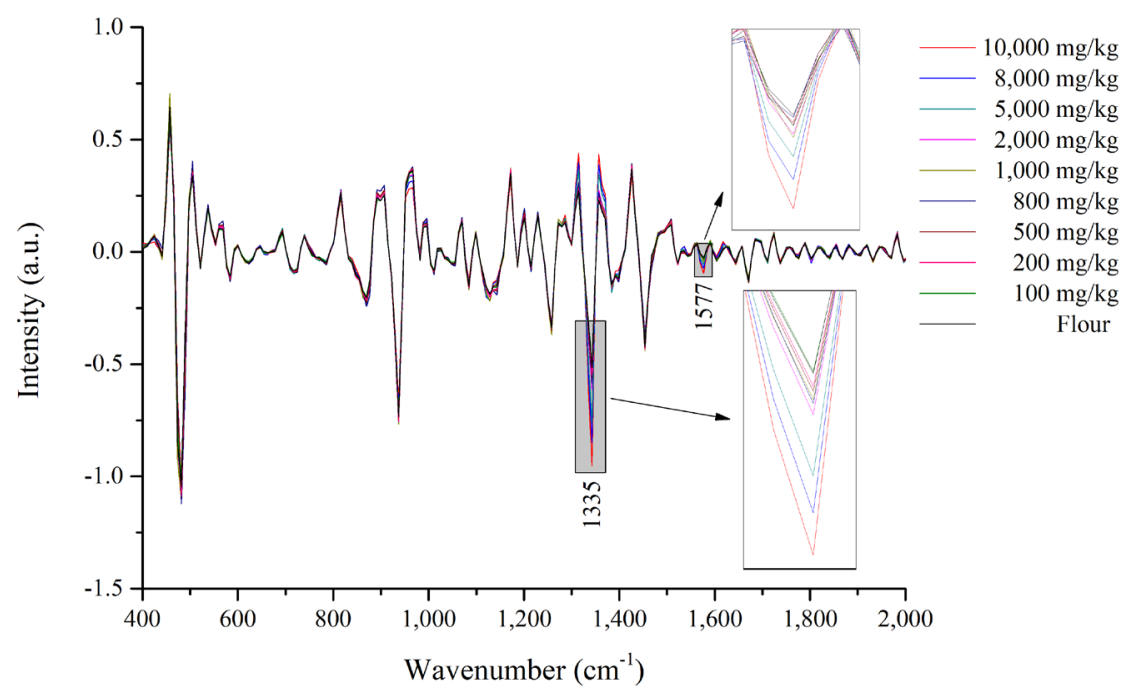

FIGURE 2. Average Raman spectra of wheat flour-azodicarbonamide mixed samples with different contents of azodicarbonamide (pretreated with second derivative).

\section{Average Raman spectra of wheat flour-ADA mixed samples}

Figure 2 shows the average Raman spectra of wheat flour-ADA mixed samples with different ADA contents in wheat flour (pretreated with the $2^{\text {nd }}$ ). The Raman spectrum of each mixed sample contained the signals from both wheat flour and ADA. The two peaks of ADA $\left(1,335\right.$ and $\left.1,577 \mathrm{~cm}^{-1}\right)$ could be observed in the spectra, but their intensity was lower than that of wheat flour, because the ADA content in mixed samples was low. The mixed sample with ADA content of $10,000 \mathrm{mg} / \mathrm{kg}$ showed the highest intensity of the Raman characteristic peaks of ADA. When the ADA content in the mixed samples decreased from 10,000 to $1,000 \mathrm{mg} / \mathrm{kg}$, the intensity of these peaks decreased continuously. The intensity of peaks recorded for samples with ADA content lower than $1,000 \mathrm{mg} / \mathrm{kg}$ was not significantly different. This indicated that the average spectrum of the mixed samples could not be used to detect ADA content in wheat flour effectively. On the other hand, Huang et al. [2016] found that the spectra corresponding to pixels can be used to effectively detect additive particles in food when detecting food additives by near-infrared hyperspectral imaging. Therefore, in the next step of our study, the Raman spectrum of each pixel in ROI was analyzed to realize the effective detection of ADA in wheat flour.

\section{Establishment of PLS model}

A total of 9 wheat flour-ADA mixed samples were prepared, and Raman images of 4 subsamples corresponding to each mixed sample were collected; then those 4 subsamples were aggregated and remixed, and divided into 4 new subsamples to collect Raman images. The process was repeated once more. The average Raman spectrum of each subsample was calculated, and a total of 108 spectra $(9 \times 4 \times 3)$ were obtained as the calibration set of the PLS model. The average Raman spectra of wheat flour and ADA (based on 4 subsamples of each) were also calculated and applied to equation (1) to obtain the 36 new Raman spectra (for 9 different ADA contents; $4 \times 9$ ) as the prediction set of the PLS model.

Table 1 shows the PLS model results established by different pretreated Raman spectra. The $R_{c}^{2}$ of the PLS model established by the original spectra was higher than that of normalized, $1^{\text {st }}$ and $2^{\text {nd }}$ derivative spectra, while the $R_{c}^{2}$ of the PLS model established by MSC and SNV spectra was higher than that of original spectra. Among all PLS models, SNV spectra had the best prediction result, and the $R_{p}^{2}$ and RMSEP values of the prediction set were 0.9212 and $0.0967 \%$, respectively. SNV was used to eliminate the interference caused by surface scattering, solid particle size, and light intensity changes on the Raman spectrum of wheat flour particles and this pretreatment method had a good correction effect [Huang et al., 2011]. The regression coefficients of the PLS model established by SNV spectra were extracted, as shown in Figure 3. Higher or lower coefficients indicate the wavenumbers that have a significant impact on the PLS model [Esquerre et al., 2011].

TABLE 1. Partial least squares model results established by different pretreated Raman spectra.

\begin{tabular}{l|ccc|c|c}
\hline \multirow{2}{*}{ Pretreatment } & \multicolumn{2}{|c|}{ Calibration set } & \multicolumn{2}{c|}{ Prediction set } & \multirow{2}{*}{ Factor } \\
\cline { 2 - 5 } & $R_{c}^{2}$ & RMSEC & $R_{p}^{2}$ & RMSEP & \\
\hline Original & 0.9934 & 0.0280 & 0.9071 & 0.1050 & 5 \\
Normalized & 0.9933 & 0.0282 & 0.9086 & 0.1042 & 3 \\
MSC & 0.9950 & 0.0244 & 0.9205 & 0.0972 & 3 \\
SNV & 0.9951 & 0.0242 & 0.9212 & 0.0967 & 3 \\
$1^{\text {st }}$ derivative & 0.9882 & 0.0374 & 0.8899 & 0.1144 & 2 \\
$2^{\text {nd }}$ derivative & 0.9930 & 0.0288 & 0.9087 & 0.1041 & 4 \\
\hline
\end{tabular}

MSC: multiplicative scatter correction; SNV: standard normal variate transformation; RMSEC: root-mean-square error of calibration set; RMSEP: root-mean-square error of prediction set. 


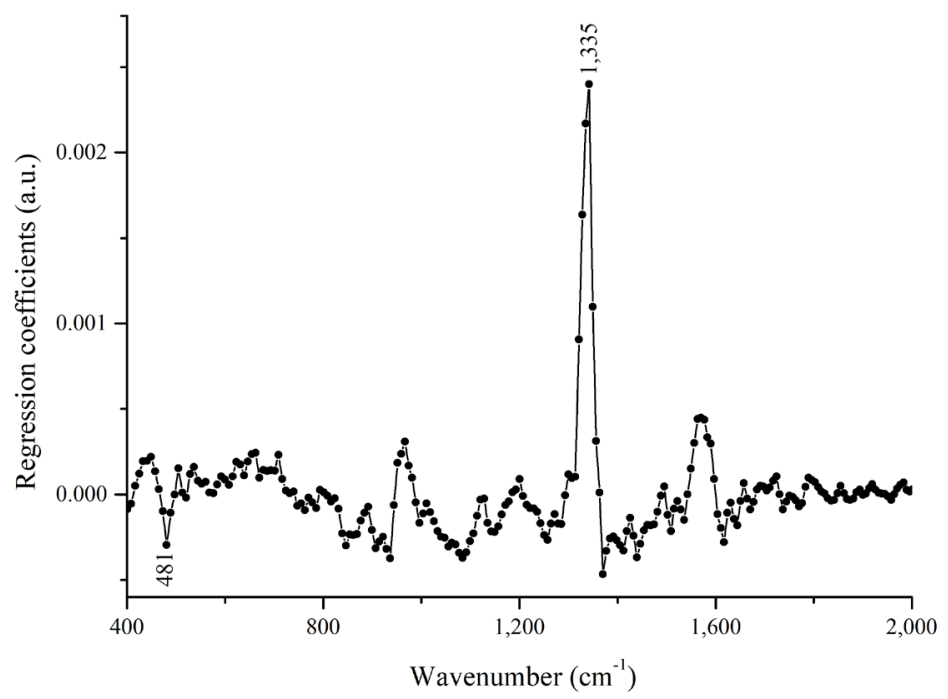

FIGURE 3. Regression coefficient of partial least squares model.

In our study, the highest regression coefficient was located at $1,335 \mathrm{~cm}^{-1}$ (Figure 3), which corresponds with the significant Raman peak of ADA (Figure 1b). The regression coefficient at $481 \mathrm{~cm}^{-1}$ (significant peak of wheat flour Raman spectrum) also had a certain impact on the PLS model.

\section{Quantitative analysis of ADA in wheat flour}

The absolute value of each regression coefficient in the PLS model was applied to the Raman image of wheat flour-ADA mixed sample, and the intensity of each pixel was calculated by equation (2) to convert the 3D Raman image into a $2 \mathrm{D}$ grayscale image. Wheat flour pixels and ADA pixels were still difficult to identify in the grayscale image of the mixed sample. Therefore, the threshold segmentation method was used to create the grayscale image as a binary image to classify wheat flour pixels and ADA pixels. The PLS model regression coefficient was also used to determine the threshold. The intensity of each pixel in the wheat flour Raman image was calculated by equation (2), and its maximum intensity was 6.9382, which was selected as the threshold. The pixels with intensity above the threshold (white pixels) were classified as ADA, whereas those with intensity below the threshold (black ones) were classified as wheat flour. No pixels classified as wheat flour were found in the pure ADA image, and no pixels classified as ADA were found in the pure wheat flour image, which indicated that the threshold can correctly classify wheat flour pixels and ADA pixels.

The number of pixels classified as ADA in 4 subsamples of each mixed sample was counted (Table 2). The values decreased with the decrease of ADA content in each subsample. The number of pixels classified as ADA in the mixed sample with the lowest ADA content was 7, 6, 9, and 8, as shown in Figure 4. This indicated that the minimum detectable content of ADA in wheat flour by this method was $100 \mathrm{mg} / \mathrm{kg}$, which was lower than the limit of detection of ADA in flour by near-infrared hyperspectral imaging $(200 \mathrm{mg} / \mathrm{kg}$ ) [Wang et al., 2018]. Differences in the number of ADA pixels among subsamples were probably caused by the random distribution of ADA particles in the mixed sample. Also, the differences in the number of ADA pixels indicate that multiple subsamples need to be used for the quantitative analysis of wheat flour-ADA mixed samples. The average number of ADA pixels of subsamples was used to establish a quantitative model (Figure 5). There was a good linear correlation between the ADA content and the average number of ADA pixels, and the coefficient of determination was 0.9858 , which indicated that the method established in this study could be used for the quantitative analysis of ADA additives in wheat flour.

Raman characteristic peak method (based on Raman imaging) was commonly used for detecting additives in food products [Dhakal et al., 2016; Qin et al., 2018; Wang et al., 2017b]. In our study, this method was used to detect ADA in wheat flour-ADA mixed samples. The single-band image corresponding to the Raman peak with the highest intensity in the ADA Raman spectrum was selected from the Raman image of the mixed sample, and the thresholding segmentation method was used to achieve the classification of wheat flour pixels and ADA pixels. The results are shown in Table 3. In all wheat flour-ADA mixed samples, the number

TABLE 2. Number of azodicarbonamide pixels classified in the four subsamples of each wheat flour-azodicarbonamide mixed sample.

\begin{tabular}{l|c|c|c|c|c}
\hline $\begin{array}{l}\text { Content } \\
(\mathrm{mg} / \mathrm{kg})\end{array}$ & $\begin{array}{c}\text { Subsample } \\
1\end{array}$ & $\begin{array}{c}\text { Subsample } \\
2\end{array}$ & $\begin{array}{c}\text { Subsample } \\
3\end{array}$ & $\begin{array}{c}\text { Subsample } \\
4\end{array}$ & Average \\
\hline 100 & 7 & 6 & 9 & 8 & 7.50 \\
200 & 24 & 23 & 28 & 25 & 25.00 \\
500 & 78 & 76 & 75 & 77 & 76.50 \\
800 & 114 & 105 & 107 & 105 & 107.75 \\
1,000 & 158 & 149 & 159 & 165 & 157.75 \\
2,000 & 316 & 310 & 308 & 319 & 313.25 \\
5,000 & 724 & 691 & 706 & 694 & 703.75 \\
8,000 & 1152 & 1097 & 1122 & 1118 & 1122.25 \\
10,000 & 1694 & 1642 & 1722 & 1653 & 1677.75 \\
\hline
\end{tabular}




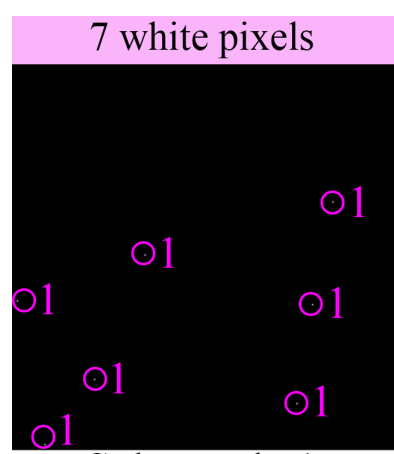

Subsample 1

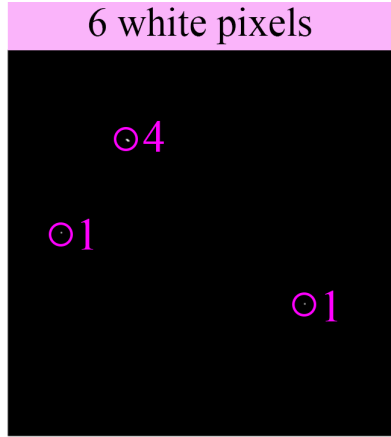

Subsample 2

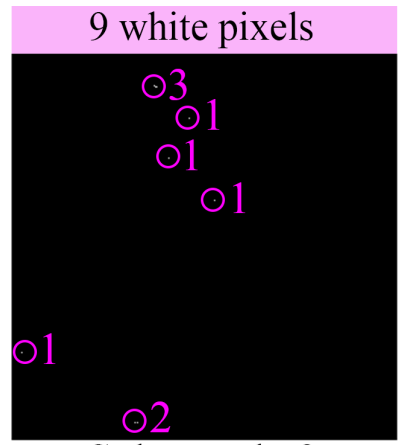

Subsample 3

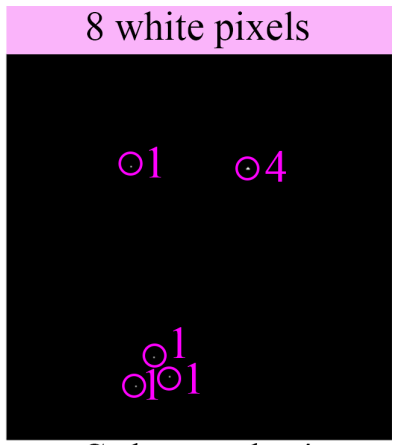

Subsample 4

FIGURE 4. Classification image of wheat flour-azodicarbonamide mixed samples with the azodicarbonamide content of $100 \mathrm{mg} / \mathrm{kg}$.

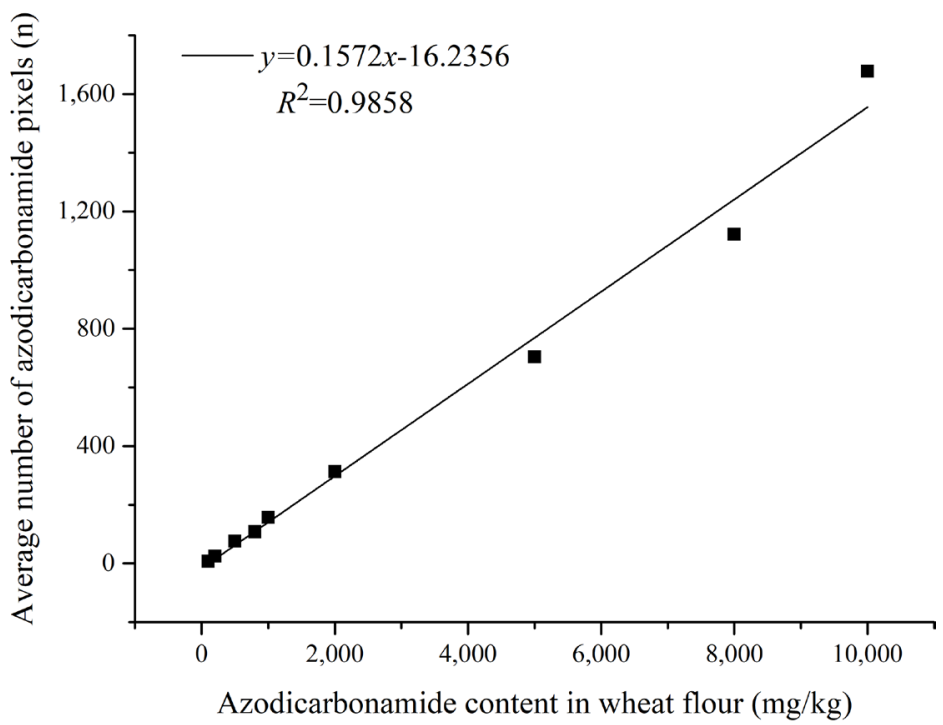

FIGURE 5. Linear relationship model between azodicarbonamide content in wheat flour and average number of azodicarbonamide pixels.

TABLE 3. Detection results of azodicarbonamide in wheat flour-azodicarbonamide mixed samples by Raman characteristic peak method.

\begin{tabular}{l|c|c|c|c|c}
\hline $\begin{array}{l}\text { Content } \\
(\mathrm{mg} / \mathrm{kg})\end{array}$ & $\begin{array}{c}\text { Subsample } \\
1\end{array}$ & $\begin{array}{c}\text { Subsample } \\
2\end{array}$ & $\begin{array}{c}\text { Subsample } \\
3\end{array}$ & $\begin{array}{c}\text { Subsample } \\
4\end{array}$ & Average \\
\hline 100 & 8 & 6 & 7 & 8 & 7.25 \\
200 & 24 & 22 & 27 & 24 & 24.25 \\
500 & 76 & 75 & 72 & 73 & 74 \\
800 & 109 & 105 & 103 & 105 & 105.5 \\
1,000 & 153 & 147 & 157 & 165 & 155.5 \\
2,000 & 309 & 307 & 303 & 316 & 308.75 \\
5,000 & 699 & 676 & 696 & 687 & 689.5 \\
8,000 & 1120 & 1080 & 1097 & 1110 & 1101.75 \\
10,000 & 1650 & 1621 & 1687 & 1641 & 1649.75 \\
\hline
\end{tabular}

of pixels classified as ADA by the PLS model regression coefficient method established in this paper (Table 2) was higher than determined with the Raman characteristic peak method. In the Raman characteristic peak method, the intensity of a single Raman peak was used as an index for determination, and the threshold was the maximum intensity of the wheat flour sample at this band. But some pixels were not detected due to their low Raman scattering intensity of ADA, which could lead to false negative results. In the PLS model regression coefficient method, the intensities of all bands of each mixed sample were used for calculation, which provided more comprehensive information and had higher detection accuracy. The linear correlation between ADA content in wheat flour-ADA mixed samples and the number of ADA pixels in Raman images proves the potential of high-throughput Raman imaging for the quantitative analysis of ADA in wheat flour. In the next research, the improvement of hardware equipment performance and spectral pretreatment methods will help to achieve lower concentration detection, and will lay a theoretical foundation for the market-oriented application of high-throughput Raman imaging.

\section{CONCLUSIONS}

In this study, the large-area detection, visual identification and non-destructive quantitative analysis of ADA additives 
in wheat flour were achieved by high-throughput Raman imaging. The average Raman spectra of wheat flour-ADA mixed samples showed that the three Raman characteristic peaks of $\operatorname{ADA}\left(1,121 ; 1,335\right.$; and $\left.1,577 \mathrm{~cm}^{-1}\right)$ were ineffective in evaluating the ADA content in wheat flour. Different spectral pretreatment methods were used for Raman spectra of mixed sample images, and the PLS model established by SNV pretreatment had a good predictive effect. All the regression coefficients of the PLS model were applied to the Raman spectra of the mixed sample images to convert them into grayscale images, but wheat flour pixels and ADA pixels were still difficult to identify. Threshold segmentation was used to classify wheat flour pixels and ADA pixels in grayscale images. The classification results showed that the minimum detection content of ADA in wheat flour was $100 \mathrm{mg} / \mathrm{kg}$, and that there was a good linear correlation between the ADA content in the mixed sample and the number of ADA pixels in the Raman image, with a determination coefficient of 0.9858 . This study provides the method for the quantitative analysis of ADA additives in wheat flour as well as a technical reference for large-scale rapid screening. This method also appears to have the potential in future applications for the analysis of contaminants in other powdered food products.

\section{RESEARCH FUNDING}

This work was supported by the Project supported by the National Natural Science Foundation of China (No. 32160417), the Science and Technology Project of Jiangxi Provincial Department of Education (No. GJJ212605), the Doctoral Research Funds of Nanchang Normal University (No. NSBSJJ2018016), and the Scientific research project of Nanchang Normal University (No. N21KJYB02).

\section{CONFLICT OF INTEREST}

Authors declare no conflict of interest.

\section{ORCID IDs}

X. Wang https://orcid.org/0000-0002-0402-6895

C. Zhao https://orcid.org/0000-0002-8641-2254

\section{REFERENCES}

1. Becalski, A., Lau, B.P.Y., Lewis, D., Seaman, S.W. (2004). Semicarbazide formation in azodicarbonamide-treated flour: A model study. Journal of Agricultural and Food Chemistry, 52(18), 5730-5734. https://doi.org/10.1021/jf0495385

2. Cebi, N., Dogan, C.E., Develioglu, A., Yayla, M.E.A., Sagdic, O. (2017). Detection of L-cysteine in wheat flour by Raman microspectroscopy combined chemometrics of HCA and PCA. Food Chemistry, 228, 116-124.

https://doi.org/10.1016/j.foodchem.2017.01.132

3. Che, W.K., Sun, L.J., Zhang, Q., Zhang, D., Ye, D.D., Tan, W.Y., Wang, L.K., Dai, C.J. (2017). Application of visible/near-infrared spectroscopy in the prediction of azodicarbonamide in wheat flour. Journal of Food Science, 82(10), 2516-2525.

https://doi.org/10.1111/1750-3841.13859
4. Chen, W., Shi, W., Li, Z., Ma, H.M., Liu, Y., Zhang, J.H., Liu, Q.J. (2011). Simple and fast fluorescence detection of benzoyl peroxide in wheat flour by $N$-methoxy rhodamine-6G spirolactam based on consecutive chemical reactions. Analytica Chimica Acta, 708(1-2), 84-88.

https://doi.org/10.1016/j.aca.2011.10.002

5. Chen, Z.Q., Chen, L., Lin, L., Wu, Y.N., Fu, F.F. (2018). A colorimetric sensor for the visual detection of azodicarbonamide in flour based on azodicarbonamide-induced anti-aggregation of gold nanoparticles. ACS Sensors, 3(10), 2145-2151.

https://doi.org/10.1021/acssensors.8b00705

6. Czaja, T., Mazurek, S., Szostak, R. (2016). Quantification of gluten in wheat flour by FT-Raman spectroscopy. Food Chemistry, $211,560-563$.

https://doi.org/10.1016/j.foodchem.2016.05.108

7. Dhakal, S., Chao, K.L., Qin, J.W., Kim, M., Chan, D.N. (2016). Raman spectral imaging for quantitative contaminant evaluation in skim milk powder. Journal of Food Measurement and Characterization, 10(2), 374-386.

https://doi.org/10.1007/s11694-016-9316-1

8. Esquerre, C., Gowen, A.A., Downey, G., O’Donnell, C.P. (2011). Selection of variables based on most stable normalised partial least squares regression coefficients in an ensemble Monte Carlo procedure. Journal of Near Infrared Spectroscopy, 19(6), 443-450. https://doi.org/10.1255/jnirs.962

9. Gao, S., Sun, L.J., Hui, G.Y., Wang, L.K., Dai, C.J., Wang, J.A. (2016). Prediction of azodicarbonamide in flour using near-infrared spectroscopy technique. Food Analytical Methods, 9(9), 2642-2648.

https://doi.org/10.1007/s12161-016-0441-6

10. Hu, J., Liu, Y.D., He, Y., Sun, X.D., Li, B. (2020). Optimization of quantitative detection model for benzoic acid in wheat flour based on CARS variable selection and THz spectroscopy. Journal of Food Measurement and Characterization, 14(5), 2549-2558. https://doi.org/10.1007/s11694-020-00501-5

11. Huang, C.W., Dai, L.K., Dong, X.F. (2011). The application of piecewise direct standardization with SNV in calibration transfer of Raman spectra. Spectroscopy and Spectral Analysis, 31(5), 1279-1282.

https://doi.org/10.3964/j.issn.1000-0593(2011)05-1279-04

12. Huang, M., Kim, M.S., Delwiche, S.R., Chao, K., Qin, J.W., Mo, C., Esquerre, C., Zhu, Q.B. (2016). Quantitative analysis of melamine in milk powders using near-infrared hyperspectral imaging, and band ratio. Journal of Food Engineering, 181, 10-19. https://doi.org/10.1016/j.jfoodeng.2016.02.017

13. Lancelot, E., Fontaine, J., Grua-Priol, J., Le-Bail, A. (2021). Effect of long-term storage conditions on wheat flour and bread baking properties. Food Chemistry, 346, art. no. 128902. https://doi.org/10.1016/j.foodchem.2020.128902

14. Li, G.L., Tang, C.H., Wang, Y., Yang, J., Wu, H.L., Chen, G., Kong, X.J., Kong, W.H., Liu, S.C., You, J.M. (2015). A rapid and sensitive method for semicarbazide screening in foodstuffs by HPLC with fluorescence detection. Food Analytical Methods, 8(7), 1804-1811. https://doi.org/10.1007/s12161-014-0063-9

15. Li, M.H., Guo, X.Y., Wang, H., Wen, Y., Yang, H.F. (2015). Rapid and label-free Raman detection of azodicarbonamide with asthma risk. Sensors and Actuators B-Chemical, 216, 535-541. https://doi.org/10.1016/j.snb.2015.04.103 
16. Li, Y., Peng, Y.K., Chao, K.L., Qin, J.W., Dhakal, S. (2019). Nondestructive rapid detection of benzoyl peroxide in flour based on Raman hyperspectral technique. Proceedings of SPIE, 11016, art. no. $110160 \mathrm{G}$. https://doi.org/10.1117/12.2517454

17. Liu, C., Liu, L., Li, L.M., Hao, C.M., Zheng, X.L., Bian, K., Zhang, J., Wang, X.X. (2015). Effects of different milling processes on whole wheat flour quality and performance in steamed bread making. LWT - Food Science and Technology, 62(1), 310-318. https://doi.org/10.1016/j.Iwt.2014.08.030

18. Lohumi, S., Kim, M.S., Qin, J.W., Cho, B.K. (2017). Raman imaging from microscopy to macroscopy: Quality and safety control of biological materials. TrAC - Trends in Analytical Chemistry, 93, 183-198.

https://doi.org/10.1016/j.trac.2017.06.002

19. Lv, J.L., Lu, Y.J., Niu, Y.G., Whent, M., Ramadan, M.F., Costa, J., Yu, L.L. (2013). Effect of genotype, environment, and their interaction on phytochemical compositions and antioxidant properties of soft winter wheat flour. Food Chemistry, 138(1), 454-462.

https://doi.org/10.1016/j.foodchem.2012.10.069

20. Noonan, G.O., Warner, C.R., Hsu, W., Begley, T.H., Perfetti, G.A., Diachenko, G.W. (2005). The determination of semicarbazide (N-Aminourea) in commercial bread products by liquid chromatography-mass spectrometry. Journal of Agricultural and Food Chemistry, 53(12), 4680-4685.

https://doi.org/10.1021/jf050480j

21. Qin J., Chao K., Kim M.S. (2010). Raman chemical imaging system for food safety and quality inspection. Transactions of the ASABE, 53(6), 1873-1882.

https://doi.org/ 10.13031/2013.35796

22. Qin, J.W., Kim, M.S., Chao, K.L., Bellato, L., Schmidt, W.F., Cho, B.K., Huang, M. (2018). Inspection of maleic anhydride in starch powder using line-scan hyperspectral Raman chemical imaging technique. International Journal of Agricultural and Biological Engineering, 11(6), 120-125.

https://doi.org/10.25165/j.ijabe.20181106.4339

23. Qin, J.W., Kim, M.S., Chao, K.L., Gonzalez, M., Cho, B.K. (2017). Quantitative detection of benzoyl peroxide in wheat flour using line-scan macroscale Raman chemical imaging. Applied Spectroscopy, 71 (11), 2469-2476.

https://doi.org/10.1177/0003702817706690

24. Sun, X.D., Zhu, K., Liu, J.B., Hu, J., Jiang, X.G., Liu, Y.D., Gong, Z.Y. (2019). Terahertz spectroscopy determination of benzoic acid additive in wheat flour by machine learning. Journal of Infrared Millimeter and Terahertz Waves, 40(4), 466-475.

https://doi.org/10.1007/s10762-019-00579-z

25. Tian, W.R., Sang, Y.X., Wang, X.H. (2014). Semicarbazide from state-of-the-art analytical methods and exposure to toxicity: a review. Food Additives and Contaminants Part A-Chemistry Analysis Control Exposure \& Risk Assessment, 31 (11), 1850-1860. https://doi.org/10.1080/19440049.2014.953012

26. Wang, X.B., Zhao, C.J., Huang, W.Q., Wang, Q.Y., Liu, C., Yang, G.Y. (2017a). Effective detection of benzoyl peroxide in flour based on parameter selection of Raman hyperspectral system. Spectroscopy Letters, 50(7), 364-369.

https://doi.org/10.1080/00387010.2017.1332649

27. Wang, X.B., Huang, W.Q., Zhao, C.J., Wang, Q.Y., Liu, C., Yang, G.Y. (2017b). Quantitative analysis of BPO additive in flour via Raman hyperspectral imaging technology. European Food Research and Technology, 243(12), 2265-2273.

https://doi.org/10.1007/s00217-017-2928-9

28. Wang, X.B., Zhao, C.J., Huang, W.Q., Wang, Q.Y., Liu, C., Yang, G.Y. (2018). Near-infrared hyperspectral imaging for detection and quantification of azodicarbonamide in flour. Journal of the Science of Food and Agriculture, 98(7), 2793-2800. https://doi.org/10.1002/jsfa.8776

29. Wang, Y., Wang, J., Xiang, L., Xi, C., Chen, D., Peng, T., Wang, G., Mu, Z. (2014). Determination of biurea in flour and its products by liquid chromatography-tandem mass spectrometry. Chinese Journal of Chromatography, 32(5), 513-518.

https://doi.org/10.3724/SP.J.1123.2013.12023

30. Wei, T.F., Li, G.K., Zhang, Z.M. (2017). Rapid determination of trace semicarbazide in flour products by high-performance liquid chromatography based on a nucleophilic substitution reaction. Journal of Separation Science, 40(9), 1993-2001.

https://doi.org/10.1002/jssc.201700045

31. Wiercigroch, E., Szafraniec, E., Czamara, K., Pacia, M.Z., Majzner, K., Kochan, K., Kaczor, A., Baranska, M., Malek, K. (2017). Raman and infrared spectroscopy of carbohydrates: A review. Spectrochimica Acta Part A - Molecular and Biomolecular Spectroscopy, 185, 317-335.

https://doi.org/10.1016/j.saa.2017.05.045

32. Xie Y.F., Li P., Zhang J., Wang H.Y., Qian H., Yao W.R. (2013). Comparative studies by IR, Raman, and surface-enhanced Raman spectroscopy of azodicarbonamide, biurea and semicarbazide hydrochloride. Spectrochimica Acta Part A - Molecular and Biomolecular Spectroscopy, 114, 80-84.

https://doi.org/10.1016/j.saa.2013.05.055

33. Yasui, A., Oishi, M., Hayafuji, C., Kobayashi, C., Shindo, T., Ozawa, H., Nakazato, M. (2016). Analysis of azodicarbonamide in wheat flour and prepared flour mixes. Food Hygiene and Safety Science, 57(5), 133-138.

https://doi.org/10.3358/shokueishi.57.133

34. Ye, J., Wang, X.H., Sang, Y.X., Liu, Q. (2011). Assessment of the determination of azodicarbonamide and its decomposition product semicarbazide: investigation of variation in flour and flour products. Journal of Agricultural and Food Chemistry, 59(17), 9313-9318.

https://doi.org/10.1021/jf201819x

35. Zhai, C., Peng, Y.K., Li, Y.Y., Zhao, J. (2017). Detection of chemical additives in food using Raman chemical imaging system. Chemical Journal of Chinese Universities, 38(3), 369-375. https://doi.org/10.7503/cjcu20160640

36. Zhang, Z.M., Chen, S., Liang, Y.Z. (2010). Baseline correction using adaptive iteratively reweighted penalized least squares. Analyst, 135(5), 1138-1146.

https://doi.org/10.1039/b922045c 